\title{
OPTIMAL CONTROL OF MULTIPLE-TIME DELAYED SYSTEMS BASED ON THE CONTROL PARAMETERIZATION METHOD
}

\author{
W. H. GUI ${ }^{1}$, X. Y. SHEN ${ }^{1}$, N. CHEN ${ }^{凶 1}$, C. H. YANG ${ }^{1}$ and L. Y. WANG ${ }^{2}$
}

(Received 22 May, 2011; revised 9 December, 2011)

\begin{abstract}
In this paper we study optimal control computation based on the control parameterization method for a class of optimal control problems involving nonlinear systems with multiple time delays subject to continuous state inequality constraints. Both the state and the control are allowed to have different time delays, and they are uncorrelated in this system. The control of the dynamical system is approximated by a piecewise constant function whose heights are taken as decision vectors. The formulae for computing the gradients of the cost and constraint functions are then derived. Based on this, a computational method for finding the optimal control is developed by utilizing the Sequential Quadratic Programming (SQP) algorithm with an active set strategy. The computational method is applied to an industrial problem arising in the purification process of zinc hydrometallurgy. Numerical simulation shows that the amount of zinc powder that is needed can be decreased significantly, thus avoiding wastage of resources.
\end{abstract}

2010 Mathematics subject classification: 49J15.

Keywords and phrases: multiple-time delayed system, control parameterization, continuous state inequality constraints, SQP algorithm.

\section{Introduction}

With the advancement of modern computers and an increasing emphasis on optimal design of large-scale dynamical systems, optimal control has become increasingly important $[3,4,6,10,12,21]$, making a major impact in industry by improving economic benefit and reducing production cost. Many real control systems involve time delays, especially multiple time delays, which can cause instability for a system and reduce the effectiveness of a control. Thus, the study of optimal control problems involving dynamical systems with multiple time delays is of practical importance.

\footnotetext{
${ }^{1}$ School of Information Science and Engineering, Central South University, Changsha, 410083,

PR China; e-mail: gwh@csu.edu.cn, shxy-0501@163.com,ningchen@csu.edu.cn, ychh@csu.edu.cn.

${ }^{2}$ School of Electrical Engineering and Renewable Energy, China Three Gorges University, Yichang, 443002, PR China; e-mail: wly@ctgu.edu.cn.

(C) Australian Mathematical Society 2012, Serial-fee code 1446-1811/2012 \$16.00
} 
In the literature, significant advancement in theory and methods has been achieved for optimal control problems involving dynamical systems with time delays. In particular, a number of computational methods have been proposed for solving these optimal control problems, and these can be classified into two main approaches: direct and indirect. The indirect approach includes the variational formulation and the dynamic programming technique. The variational formulation is based on the Pontryagin maximum principle. This is a set of necessary conditions for optimality from which a two- or multi-point boundary value problem is obtained. However, such a boundary value problem is usually very difficult to solve [4, 17, 18]. The dynamic programming technique is based on Bellman's principle of optimality, which is surprisingly clear and intuitive yet has made a tremendous contribution in a wide range of applications. Its application in optimal control theory has led to the invention of the famous Hamilton-Jacobi-Bellman (HJB) partial differential equation, whose solution can be used to construct a nonlinear optimal feedback control law [4, 14]. However, it is very difficult to obtain the analytical solution [1] of the HJB equation. The method proposed by Chachuat et al. [4] is for a relatively simple system with a single time delay, while the method reported by Basin and Rodriguez-Gonzalez [1] is for a nonlinear system with multiple time delays appearing in the control input.

The algorithms developed using the direct approach include control parameterization [7, 8, 10, 12, 16, 17, 19, 21-23], iterative dynamic programming [9], orthogonal collocation [2] and intelligent optimization [11]. Iterative dynamic programming has been used for the optimal control of chemical processes, but it appears that this method is computationally very costly. For orthogonal collocation, the dynamic optimization problem is approximated as a constrained nonlinear programming problem (NLP) with equality and inequality constraints. The constrained NLP is then solved using a strategy that tries to achieve simultaneously the convergence and optimization of the algebraic model. However, the constrained NLP obtained from orthogonal collocation is of very large scale for an acceptable accuracy, and hence it can be very time consuming to solve such a constrained NLP. Some of the methods developed based on the idea of intelligent optimization (for example, the ant colony framework) are computationally quite efficient. However, these methods are heuristic in nature, and hence they often encounter instability in the process of finding a solution.

For the control parameterization technique, the main idea is to divide the time interval into several subintervals. The control is then approximated by a piecewise constant function with possible discontinuities at the partition points, which are called switching times. The heights of the piecewise constant functions are regarded as the decision variables. Teo et al. [17] consider a class of optimal control problems subject to equality constraints and inequality constraints involving state and control variables in canonical form. For optimal control problems subject to continuous inequality constraints on the state variables, the constraint transcription method [17] can be used to approximate the continuous inequality constraints by a sequence of inequality constraints in canonical form [17]. Thus, by using the control parameterization technique, the optimal control problem can be approximated as a 
sequence of parameter optimization problems. Each of these parameter optimization problems can be considered as a constrained NLP and hence can be solved by using a gradient-based optimization method. For the control parameterization technique, it is intuitively clear that better results would be obtained if the switching times were also regarded as decision variables. For this reason, a novel transformation, which is now known as the time scaling transform, was proposed [19]. With this transform, the transformed optimal control problems become of the same form as those considered by Teo et al. [17], and hence can be solved in the same way. Loxton et al. [8] use the time scaling transform [19] to extend the results obtained by Teo et al. [17] to the case where the continuous inequality constraints involve not only the state but also the control.

A dynamic model of an aluminium trihydroxide batch crystallization is considered by Rehbock and Livk [12]. The process model, which takes into account kinetics of nucleation, growth and agglomeration, is based on the mass balance of the process and the population balance of the dispersed crystals. A computational algorithm based on the control parameterization technique and the time scaling transform are applied to the model to optimize various performance criteria.

Wu and Teo [23] develop a global computational approach for solving a class of optimal control problems governed by impulsive dynamical systems and subject to continuous state inequality constraints. By using the time scaling transform [19], it is shown that this problem is equivalent to an optimal control problem governed by ordinary differential equations with periodic boundary conditions and subject to a set of continuous state inequality constraints. For this equivalent optimal control problem, the constraint transcription method [17] is used in conjunction with a penalty function (similar to the approach of Teo et al. [20]) to construct an appended new cost function. This leads to a sequence of approximate optimal control problems only subject to periodic boundary conditions. Each of these approximate problems can be solved as an optimization problem using gradient-based optimization techniques. A filled function method is then introduced to supplement the gradient-based optimization method to find a better solution.

The control parameterization technique [17] is used and an optimization algorithm based on an improved particle swarm optimization algorithm is constructed by Liu et al. [6]. Moreover, the optimal control problem can be transformed into a discretevalued optimal control problem [24]. The time scaling transform [19] is used to convert this discrete-valued optimal control problem into an equivalent constrained optimal parameter selection problem, which can be solved by existing optimal control techniques. Furthermore, based on the work of Liu et al. [17], the class of optimal control problems involves fixed multiple characteristic times in its cost and constraint functions. For the case when the characteristic time points are free, a computational method is developed by Loxton et al. [7] by novel application of the time scaling transform [19].

The case of multiple state and control time delays is not considered in any of the papers mentioned above. Wang et al. [21] consider a class of optimal control problems 
involving single-time delayed dynamical systems with multiple characteristic time points appearing in the cost and constraint functions. However, in this paper, we consider a class of optimal control problems involving multiple time delays and subject to continuous state inequality constraints. This problem contains the general cases because of the multiple time delays. The formulation of this time delayed optimal control problem is motivated by an industrial problem arising in the purification process of zinc hydrometallurgy. We develop a computational method to solve this multiple-time delayed optimal control problem based on the control parameterization technique. The method obtained is then applied to control optimally the chemical reaction system involving the purification process of zinc sulphate electrolyte so that wastage of resources is avoided.

\section{Problem description}

Consider a process described by the following system of multiple-time delayed differential equations defined on the fixed time interval $(0, T]$ :

$$
\frac{d \mathbf{x}(t)}{d t}=\mathbf{f}\left(t, \mathbf{x}(t), \mathbf{x}\left(t-\alpha_{1}\right), \ldots, \mathbf{x}\left(t-\alpha_{m_{1}}\right), \mathbf{u}(t), \mathbf{u}\left(t-\beta_{1}\right), \ldots, \mathbf{u}\left(t-\beta_{m_{2}}\right)\right),
$$

where $\mathbf{x}=\left[x_{1}, \ldots, x_{n}\right]^{\mathrm{T}} \in \mathbb{R}^{n}$ and $\mathbf{u}=\left[u_{1}, \ldots, u_{r}\right]^{\mathrm{T}} \in \mathbb{R}^{r}$ are the state and control vectors, respectively, $\mathbf{f}=\left[f_{1}, \ldots, f_{n}\right]^{\mathrm{T}} \in \mathbb{R}^{n}$, the $\alpha_{k}$ are the state time delays satisfying $0<\alpha_{k}<T, k=1,2, \ldots, m_{1}$, and the $\beta_{k}$ are the control time delays satisfying $0<\beta_{k}<T, k=1,2, \ldots, m_{2}$. Let

$$
0<\alpha_{1} \leq \cdots \leq \alpha_{m_{1}}<T, \quad 0<\beta_{1} \leq \cdots \leq \beta_{m_{2}}<T,
$$

and, moreover, let $\alpha_{1}, \ldots, \alpha_{m_{1}}, \beta_{1}, \ldots, \beta_{m_{2}}$ be ordered increasing from small to large. Then let $h_{i}, i=1,2, \ldots, l$, denote the ordered time delays, where $1 \leq l \leq m_{1}+m_{2}$ (that is, $h_{1}$ is the minimum delay and $h_{l}$ is the maximum delay). For the case when $l=m_{1}+m_{2}$, this means that the values $\alpha_{1}, \ldots, \alpha_{m_{1}}, \beta_{1}, \ldots, \beta_{m_{2}}$ are all different.

The initial condition for the differential equation (2.1) is

$$
\mathbf{x}(t)=\varphi(t), \quad \mathbf{u}(t)=\gamma(t), \quad t \in\left[-h_{l}, 0\right), \quad \mathbf{x}(0)=\mathbf{x}^{0},
$$

where $\varphi(t)=\left[\varphi_{1}(t), \ldots, \varphi_{n}(t)\right]^{\mathrm{T}}$ and $\gamma(t)=\left[\gamma_{1}(t), \ldots, \gamma_{r}(t)\right]^{\mathrm{T}}$ are given piecewise continuous functions from $\left[-h_{l}, 0\right)$ into $\mathbb{R}^{n}$ and $\mathbb{R}^{r}$, respectively, and $\mathbf{x}^{0}$ is a given vector in $\mathbb{R}^{n}$. We now state the canonical optimal control problem with multiple time delays.

Problem (P1). Given the system (2.1)-(2.2), find a control $\mathbf{u} \in \mathcal{U}$ such that the cost function

$$
\begin{aligned}
& g_{0}(\mathbf{u})= \Phi_{0}(\mathbf{x}(T \mid \mathbf{u}))+\int_{0}^{T} \mathcal{L}_{0}\left(t, \mathbf{x}(t \mid \mathbf{u}), \mathbf{x}\left(t-\alpha_{1} \mid \mathbf{u}\right), \ldots, \mathbf{x}\left(t-\alpha_{m_{1}} \mid \mathbf{u}\right),\right. \\
&\left.\mathbf{u}(t), \mathbf{u}\left(t-\beta_{1}\right), \ldots, \mathbf{u}\left(t-\beta_{m_{2}}\right)\right) d t
\end{aligned}
$$


is minimized subject to the canonical inequality constraints

$$
\begin{gathered}
g_{i}(\mathbf{u})=\Phi_{i}(\mathbf{x}(T \mid \mathbf{u}))+\int_{0}^{T} \mathcal{L}_{i}\left(t, \mathbf{x}(t \mid \mathbf{u}), \mathbf{x}\left(t-\alpha_{1} \mid \mathbf{u}\right), \ldots, \mathbf{x}\left(t-\alpha_{m_{1}} \mid \mathbf{u}\right),\right. \\
\left.\mathbf{u}(t), \mathbf{u}\left(t-\beta_{1}\right), \ldots, \mathbf{u}\left(t-\beta_{m_{2}}\right)\right) d t \geq 0
\end{gathered}
$$

$i=1, \ldots, N$, where $\Phi_{i}$ and $\mathcal{L}_{i}, i=0,1, \ldots, N$, are given real-valued functions. Here $\mathcal{U}$ is the class of admissible controls [17].

Let $\mathcal{F}$ be the set that consists of all of the elements of $\mathcal{U}$ such that the constraints (2.4) are satisfied. Elements of $\mathcal{F}$ are called feasible controls and $\mathcal{F}$ is called the class of feasible controls. We assume throughout that the following conditions are satisfied:

(A1) We have

$$
\begin{aligned}
& \mathbf{f}:[0, T] \times \mathbb{R}^{n+m_{1} n} \times \mathbb{R}^{r+m_{2} r} \rightarrow \mathbb{R}^{n}, \\
& \varphi:\left[-h_{l}, 0\right) \rightarrow \mathbb{R}^{n}, \quad \gamma:\left[-h_{l}, 0\right) \rightarrow \mathbb{R}^{r}, \\
& \Phi_{i}: \mathbb{R}^{n} \rightarrow \mathbb{R}, \quad i=0,1, \ldots, N, \\
& \mathcal{L}_{i}:[0, T] \times \mathbb{R}^{n+m_{1} n} \times \mathbb{R}^{r+m_{2} r} \rightarrow \mathbb{R}, \quad i=0,1, \ldots, N .
\end{aligned}
$$

(A2) For each $i=0,1, \ldots, N$ and any compact subset $V \subset \mathbb{R}^{r}$, there exists a positive constant $K$ such that

$$
\begin{aligned}
& \left|\mathbf{f}\left(t, \mathbf{x}, \mathbf{y}_{1}, \ldots, \mathbf{y}_{m_{1}}, \mathbf{u}, \mathbf{v}_{1}, \ldots, \mathbf{v}_{m_{2}}\right)\right| \leq K\left(1+|\mathbf{x}|+\left|\mathbf{y}_{1}\right|+\cdots+\left|\mathbf{y}_{m_{1}}\right|\right) \\
& \left|\mathcal{L}_{i}\left(t, \mathbf{x}, \mathbf{y}_{1}, \ldots, \mathbf{y}_{m_{1}}, \mathbf{u}, \mathbf{v}_{1}, \ldots, \mathbf{v}_{m_{2}}\right)\right| \leq K\left(1+|\mathbf{x}|+\left|\mathbf{y}_{1}\right|+\cdots+\left|\mathbf{y}_{m_{1}}\right|\right)
\end{aligned}
$$

for all $\left(t, \mathbf{x}, \mathbf{y}_{1}, \ldots, \mathbf{y}_{m_{1}}, \mathbf{u}, \mathbf{v}_{1}, \ldots, \mathbf{v}_{m_{2}}\right) \in[0, T] \times \mathbb{R}^{n+m_{1} n} \times V \times V^{m_{2}}$.

(A3) For each $i=0,1, \ldots, N$,

$$
\mathbf{f}\left(t, \mathbf{x}, \mathbf{y}_{1}, \ldots, \mathbf{y}_{m_{1}}, \mathbf{u}, \mathbf{v}_{1}, \ldots, \mathbf{v}_{m_{2}}\right) \quad \text { and } \quad \mathcal{L}_{i}\left(t, \mathbf{x}, \mathbf{y}_{1}, \ldots, \mathbf{y}_{m_{1}}, \mathbf{u}, \mathbf{v}_{1}, \ldots, \mathbf{v}_{m_{2}}\right)
$$

are piecewise continuous on $[0, T]$ for each $\left(\mathbf{x}, \mathbf{y}_{1}, \ldots, \mathbf{y}_{m_{1}}, \mathbf{u}, \mathbf{v}_{1}, \ldots, \mathbf{v}_{m_{2}}\right) \in$ $\mathbb{R}^{n+m_{1} n} \times \mathbb{R}^{r+m_{2} r}$, and are continuously differentiable with respect to each of the components $\mathbf{x}, \mathbf{y}_{1}, \ldots, \mathbf{y}_{m_{1}}, \mathbf{u}, \mathbf{v}_{1}, \ldots, \mathbf{v}_{m_{2}}$ for each fixed $t \in[0, T]$.

(A4) For each $i=0,1, \ldots, N, \Phi_{i}$ is continuously differentiable with respect to $\mathbf{x}$. Moreover, $\varphi$ and $\gamma$ are piecewise continuous on $\left[-h_{l}, 0\right)$.

In practice, physical constraints are often not expressed by the canonical constraints defined by (2.4). Instead, they are described by continuous state inequality constraints given by

$$
h_{i}(t, \mathbf{x}(t \mid \mathbf{u})) \geq 0 \quad \text { for all } t \in[0, T], i=1, \ldots, N,
$$

where the $h_{i}$ are real-valued functions defined on $[0, T] \times \mathbb{R}^{n}$. We now describe the corresponding optimal control problem. 
Problem (P2). Given the multiple-time delayed system (2.1)-(2.2), find a control $\mathbf{u} \in \mathcal{U}$ such that the cost function (2.3) is minimized subject to the continuous state inequality constraints (2.5).

Problem (P2) cannot be solved as such due to the presence of the continuous state inequality constraints. However, these continuous inequality constraints can be approximated by a sequence of inequality constraints in canonical form using the constraint transcription method introduced by Teo et al. [17, 18].

For each $i=1, \ldots, N$, define

$$
g_{i, \varepsilon}(\mathbf{u})=\delta+\int_{0}^{T} \mathcal{L}_{i, \varepsilon}(t, \mathbf{x}(t \mid \mathbf{u})) d t \geq 0,
$$

where

$$
\mathcal{L}_{i, \varepsilon}(t, \mathbf{x}(t \mid \mathbf{u}))= \begin{cases}h_{i} & \text { if } h_{i}<-\varepsilon \\ -\left(h_{i}-\varepsilon\right)^{2} / 4 \varepsilon & \text { if }-\varepsilon \leq h_{i} \leq \varepsilon \\ 0 & \text { if } h_{i}>\varepsilon\end{cases}
$$

while $\varepsilon>0$ is an adjustable parameter controlling the accuracy of the approximation and $\delta>0$ is an adjustable parameter controlling the feasibility of the constraint (2.5). Thus, Problem (P2) is approximated by a sequence of optimal control problems defined below.

Problem $(\mathrm{P} 2(\varepsilon, \delta))$. Given the multiple-time delayed system (2.1)-(2.2), find a control $\mathbf{u} \in \mathcal{U}$ such that the cost function (2.3) is minimized subject to the canonical inequality constraint (2.6).

For each $\varepsilon>0$ and $\delta>0$, Problem $(\mathrm{P} 2(\varepsilon, \delta))$ is in the form of Problem (P1). It is known that for any $\varepsilon>0$, if $\delta>0$ is chosen sufficiently small, the solution of Problem $(\mathrm{P} 2(\varepsilon, \delta))$ will satisfy the continuous state inequality constraint $(2.5)$. The proof of this result is given by Teo et al. [17, Ch. 8].

\section{An algorithm for multiple-time delayed systems}

The control parameterization method is now used to construct a numerical algorithm to solve Problem $\mathrm{P} 2(\varepsilon, \delta)$. The gradient formulae for the cost and constraint functions with respect to the control $\mathbf{u}$ are derived. Based on this, the optimization software NLPQLP [15], which is a Fortran implementation of the SQP algorithm, can be utilized to construct a numerical algorithm to solve Problem $(\mathrm{P} 2(\varepsilon, \delta))$. The derivations of these gradient formulae are given below.

3.1. Gradient formulae For each $i=0,1, \ldots, N$, consider the following system, which is known as the corresponding co-state system:

$$
\frac{d \lambda^{i}(t)}{d t}=-\left[\frac{\partial H_{i}\left(t,\left[\mathbf{x}, \mathbf{y}_{1}, \mathbf{z}_{1}, \ldots, \mathbf{y}_{m_{1}}, \mathbf{z}_{m_{1}}, \mathbf{u}, \mathbf{v}_{1}, \mathbf{w}_{1}, \ldots, \mathbf{v}_{m_{2}}, \mathbf{w}_{m_{2}}, \lambda^{i}\right](t)\right)}{\partial \mathbf{x}}\right]^{\mathrm{T}},
$$


with boundary conditions

$$
\begin{aligned}
\lambda^{i}(T) & =\left[\frac{\partial \Phi_{i}(\mathbf{x}(T \mid \mathbf{u}))}{\partial \mathbf{x}}\right]^{\mathrm{T}}, \\
\lambda^{i}(t) & =0, \quad t>T,
\end{aligned}
$$

where

$$
\begin{aligned}
\mathbf{y}_{1}(t) & =\mathbf{x}\left(t-\alpha_{1} \mid \mathbf{u}\right), \ldots, \mathbf{y}_{m_{1}}(t)=\mathbf{x}\left(t-\alpha_{m_{1}} \mid \mathbf{u}\right), \\
\mathbf{z}_{1}(t) & =\mathbf{x}\left(t+\alpha_{1} \mid \mathbf{u}\right), \ldots, \mathbf{z}_{m_{1}}(t)=\mathbf{x}\left(t+\alpha_{m_{1}} \mid \mathbf{u}\right), \\
\mathbf{v}_{1}(t) & =\mathbf{u}\left(t-\beta_{1}\right), \ldots, \mathbf{v}_{m_{2}}(t)=\mathbf{u}\left(t-\beta_{m_{2}}\right), \\
\mathbf{w}_{1}(t) & =\mathbf{u}\left(t+\beta_{1}\right), \ldots, \mathbf{w}_{m_{2}}(t)=\mathbf{u}\left(t+\beta_{m_{2}}\right), \\
\lambda_{\alpha_{1}}^{i}(t) & =\lambda^{i}\left(t+\alpha_{1}\right), \ldots, \lambda_{\alpha_{m_{1}}}^{i}(t)=\lambda^{i}\left(t+\alpha_{m_{1}}\right), \\
\lambda_{\beta_{1}}^{i}(t) & =\lambda^{i}\left(t+\beta_{1}\right), \ldots, \lambda_{\beta_{m_{2}}}^{i}(t)=\lambda^{i}\left(t+\beta_{m_{2}}\right)
\end{aligned}
$$

and $H_{i}$ is the corresponding Hamiltonian function,

$$
\begin{aligned}
H_{i}\left(t, \mathbf{x}, \mathbf{y}_{1}, \ldots, \mathbf{y}_{m_{1}}, \mathbf{z}_{1}, \ldots, \mathbf{z}_{m_{1}}, \mathbf{u}, \mathbf{v}_{1}, \ldots, \mathbf{v}_{m_{2}},\right. \\
\left.\mathbf{w}_{1}, \ldots, \mathbf{w}_{m_{2}}, \lambda^{i}, \lambda_{\alpha_{1}}^{i}, \ldots, \lambda_{\alpha_{m_{1}}}^{i}, \lambda_{\beta_{1}}^{i}, \ldots, \lambda_{\beta_{m_{2}}}^{i}\right) \\
=\mathcal{L}_{i}\left(t, \mathbf{x}, \mathbf{y}_{1}, \ldots, \mathbf{y}_{m_{1}}, \mathbf{u}, \mathbf{v}_{1}, \ldots, \mathbf{v}_{m_{2}}\right) \\
\quad+\sum_{k=1}^{m_{1}} \mathcal{L}_{i}\left(t+\alpha_{k},\left[\mathbf{x}, \mathbf{y}_{1}, \ldots, \mathbf{y}_{m_{1}}, \mathbf{u}, \mathbf{v}_{1}, \ldots, \mathbf{v}_{m_{2}}\right]\left(t+\alpha_{k}\right)\right) e\left(T-t-\alpha_{k}\right) \\
\quad+\sum_{k=1}^{m_{2}} \mathcal{L}_{i}\left(t+\beta_{k},\left[\mathbf{x}, \mathbf{y}_{1}, \ldots, \mathbf{y}_{m_{1}}, \mathbf{u}, \mathbf{v}_{1}, \ldots, \mathbf{v}_{m_{2}}\right]\left(t+\beta_{k}\right)\right) e\left(T-t-\beta_{k}\right) \\
\quad+\left(\lambda^{i}\right)^{\mathrm{T}} \mathbf{f}\left(t, \mathbf{x}, \mathbf{y}_{1}, \ldots, \mathbf{y}_{m_{1}}, \mathbf{u}, \mathbf{v}_{1}, \ldots, \mathbf{v}_{m_{2}}\right) \\
\quad+\sum_{k=1}^{m_{1}}\left(\lambda_{\alpha_{k}}^{i}\right)^{\mathrm{T}} \mathbf{f}\left(t+\alpha_{k},\left[\mathbf{x}, \mathbf{y}_{1}, \ldots, \mathbf{y}_{m_{1}}, \mathbf{u}, \mathbf{v}_{1}, \ldots, \mathbf{v}_{m_{2}}\right]\left(t+\alpha_{k}\right)\right) e\left(T-t-\alpha_{k}\right) \\
\quad+\sum_{k=1}^{m_{2}}\left(\lambda_{\beta_{k}}^{i}\right)^{\mathrm{T}} \mathbf{f}\left(t+\beta_{k},\left[\mathbf{x}, \mathbf{y}_{1}, \ldots, \mathbf{y}_{m_{1}}, \mathbf{u}, \mathbf{v}_{1}, \ldots, \mathbf{v}_{m_{2}}\right]\left(t+\beta_{k}\right)\right) e\left(T-t-\beta_{k}\right)
\end{aligned}
$$

where $e(\cdot)$ is the unit step function.

Furthermore, we set

$$
\begin{array}{r}
\mathbf{z}_{1}(t)=0, \ldots, \mathbf{z}_{m_{1}}(t)=0, \quad i=1, \ldots, m_{1} \quad \text { for all } t \in\left[T-\alpha_{i}, T\right] \\
\mathbf{w}_{1}(t)=0, \ldots, \mathbf{w}_{m_{2}}(t)=0, \quad i=1, \ldots, m_{2} \quad \text { for all } t \in\left[T-\beta_{i}, T\right] .
\end{array}
$$

Remark 3.1. To solve the dynamical system (2.1)-(2.2), we divide the interval $[0, T]$ into $\left[0, h_{l}\right],\left[k h_{l},(k+1) h_{l}\right], k=1, \ldots, \eta-1,\left[\eta h_{l}, T\right]$, where $\eta=\operatorname{Int}\left(T / h_{l}\right)$. Then we still need to subdivide each of the subintervals into $l$ further subintervals according to 
different time delays. For example, subdivide the interval $\left[0, h_{l}\right]$ into $\left[0, h_{1}\right],\left[h_{j}, h_{j+1}\right]$, $j=1, \ldots, l-1$; the rest of the subintervals can be subdivided in the same manner. Therefore, the dynamical system (2.1)-(2.2) can be solved forward in time on each of these shorter intervals successively as ordinary differential equations.

Remark 3.2. To solve the co-state system (3.1)-(3.3), we subdivide the interval $[0, T]$ into $\left[T-h_{l}, T\right],\left[T-(k+1) h_{l}, T-k h_{l}\right], k=1, \ldots, \eta-1,\left[0, T-\eta h_{l}\right]$, where $\eta=\operatorname{Int}\left(T / h_{l}\right)$. Similarly, we need to subdivide each of the subintervals into $l$ further subintervals according to different time delays. For example, subdivide the interval $\left[T-h_{l}, T\right]$ into $\left[T-h_{1}, T\right],\left[T-h_{j}, T-h_{j-1}\right], j=2,3, \ldots, l$; the rest of the subintervals can be subdivided in the same manner. Therefore, the co-state system (3.1)-(3.3) can be solved backward in time on each of these shorter intervals successively as ordinary differential equations.

Theorem 3.3. Let $\mathbf{u}$ be any control in $\mathcal{U}$, and let $\Delta \mathbf{u}$ be any bounded measurable function defined on $\left[-h_{l}, T\right]$ such that $\Delta \mathbf{u}(t) \in \mathbb{R}^{r}$ for all $t \in[0, T]$ and $\Delta \mathbf{u}(t)=0$ for all $t \in\left[-h_{l}, 0\right)$. Then, for each $i=0,1, \ldots, N$, the directional derivative for the function $g_{i}$ is

$$
\begin{aligned}
\Delta g_{i}(\mathbf{u}) & =\lim _{\varepsilon \rightarrow 0}\left\{\frac{g_{i}(\mathbf{u}+\varepsilon \Delta \mathbf{u})-g_{i}(\mathbf{u})}{\varepsilon}\right\}=\left.\frac{d g_{i}(\mathbf{u}+\varepsilon \Delta \mathbf{u})}{d \varepsilon}\right|_{\varepsilon=0} \\
& =\frac{\partial g_{i}(\mathbf{u})}{\partial \mathbf{u}} \Delta \mathbf{u}=\int_{0}^{T} \frac{\partial \bar{H}_{i}(t)}{\partial \mathbf{u}} \Delta \mathbf{u}(t) d t
\end{aligned}
$$

where

$$
\begin{array}{r}
\bar{H}_{i}(t)=H_{i}\left(t, \mathbf{x}, \mathbf{y}_{1}, \ldots, \mathbf{y}_{m_{1}}, \mathbf{z}_{1}, \ldots, \mathbf{z}_{m_{1}}, \mathbf{u}, \mathbf{v}_{1}, \ldots, \mathbf{v}_{m_{2}},\right. \\
\left.\mathbf{w}_{1}, \ldots, \mathbf{w}_{m_{2}}, \lambda^{i}, \lambda_{\alpha_{1}}^{i}, \ldots, \lambda_{\alpha_{m_{1}}}^{i}, \lambda_{\beta_{1}}^{i}, \ldots, \lambda_{\beta_{m_{2}}}^{i}\right)
\end{array}
$$

Proof. Define

$$
\mathbf{u}(t ; \varepsilon)=\mathbf{u}(t)+\varepsilon \Delta \mathbf{u}(t) .
$$

For brevity, let $\mathbf{x}(\cdot)$ and $\mathbf{x}(\cdot ; \varepsilon)$ denote the solutions of the system (2.1)-(2.2) corresponding to $\mathbf{u}(\cdot)$ and $\mathbf{u}(\cdot ; \varepsilon)$, respectively. Furthermore, let

$$
\begin{array}{ll}
\mathbf{y}_{1}(\cdot), \ldots, \mathbf{y}_{m_{1}}(\cdot), & \mathbf{z}_{1}(\cdot), \ldots, \mathbf{z}_{m_{1}}(\cdot) \\
\mathbf{v}_{1}(\cdot), \ldots, \mathbf{v}_{m_{2}}(\cdot), & \mathbf{w}_{1}(\cdot), \ldots, \mathbf{w}_{m_{2}}(\cdot)
\end{array}
$$

and

$$
\begin{array}{ll}
\mathbf{y}_{1}(\cdot ; \varepsilon), \ldots, \mathbf{y}_{m_{1}}(\cdot ; \varepsilon), & \mathbf{z}_{1}(\cdot ; \varepsilon), \ldots, \mathbf{z}_{m_{1}}(\cdot ; \varepsilon), \\
\mathbf{v}_{1}(\cdot ; \varepsilon), \ldots, \mathbf{v}_{m_{2}}(\cdot ; \varepsilon), & \mathbf{w}_{1}(\cdot ; \varepsilon), \ldots, \mathbf{w}_{m_{2}}(\cdot ; \varepsilon)
\end{array}
$$

be defined from $\mathbf{x}(\cdot), \mathbf{u}(\cdot)$ and $\mathbf{x}(\cdot ; \varepsilon), \mathbf{u}(\cdot ; \varepsilon)$, respectively. 
From (2.1),

$$
\begin{gathered}
\mathbf{x}(t)=\mathbf{x}^{0}+\int_{0}^{t} \mathbf{f}\left(s,\left[\mathbf{x}, \mathbf{y}_{1}, \ldots, \mathbf{y}_{m_{1}}, \mathbf{u}, \mathbf{v}_{1}, \ldots, \mathbf{v}_{m_{2}}\right](s)\right) d s \\
\mathbf{x}(t ; \varepsilon)=\mathbf{x}^{0}+\int_{0}^{t} \mathbf{f}\left(s,\left[\mathbf{x}, \mathbf{y}_{1}, \ldots, \mathbf{y}_{m_{1}}, \mathbf{u}, \mathbf{v}_{1}, \ldots, \mathbf{v}_{m_{2}}\right](s ; \varepsilon)\right) d s .
\end{gathered}
$$

Thus, using Taylor's theorem, it follows that

$$
\begin{aligned}
& \Delta \mathbf{x}(t)=\lim _{\varepsilon \rightarrow 0}\left[\frac{\mathbf{x}(t ; \varepsilon)-\mathbf{x}(t)}{\varepsilon}\right] \\
&=\int_{0}^{t}\left[\frac{\partial \mathbf{f}\left(s,\left[\mathbf{x}, \mathbf{y}_{1}, \ldots, \mathbf{y}_{m_{1}}, \mathbf{u}, \mathbf{v}_{1}, \ldots, \mathbf{v}_{m_{2}}\right](s)\right)}{\partial \mathbf{x}} \Delta \mathbf{x}(s)\right. \\
&+ \frac{\partial \mathbf{f}\left(s,\left[\mathbf{x}, \mathbf{y}_{1}, \ldots, \mathbf{y}_{m_{1}}, \mathbf{u}, \mathbf{v}_{1}, \ldots, \mathbf{v}_{m_{2}}\right](s)\right)}{\partial \mathbf{y}_{1}} \Delta \mathbf{y}_{1}(s) \\
&+\cdots+\frac{\partial \mathbf{f}\left(s,\left[\mathbf{x}, \mathbf{y}_{1}, \ldots, \mathbf{y}_{m_{1}}, \mathbf{u}, \mathbf{v}_{1}, \ldots, \mathbf{v}_{m_{2}}\right](s)\right)}{\partial \mathbf{y}_{m_{1}}} \Delta \mathbf{y}_{m_{1}}(s) \\
&+\frac{\partial \mathbf{f}\left(s,\left[\mathbf{x}, \mathbf{y}_{1}, \ldots, \mathbf{y}_{m_{1}}, \mathbf{u}, \mathbf{v}_{1}, \ldots, \mathbf{v}_{m_{2}}\right](s)\right)}{\partial \mathbf{u}} \Delta \mathbf{u}(s) \\
&+\frac{\partial \mathbf{f}\left(s,\left[\mathbf{x}, \mathbf{y}_{1}, \ldots, \mathbf{y}_{m_{1}}, \mathbf{u}, \mathbf{v}_{1}, \ldots, \mathbf{v}_{m_{2}}\right](s)\right)}{\partial \mathbf{v}_{1}} \Delta \mathbf{v}_{1}(s) \\
&\left.+\cdots+\frac{\partial \mathbf{f}\left(s,\left[\mathbf{x}, \mathbf{y}_{1}, \ldots, \mathbf{y}_{m_{1}}, \mathbf{u}, \mathbf{v}_{1}, \ldots, \mathbf{v}_{m_{2}}\right](s)\right)}{\partial \mathbf{v}_{m_{2}}} \Delta \mathbf{v}_{m_{2}}(s)\right] d s
\end{aligned}
$$

The quantity $\Delta \mathbf{x}(t)$ satisfies the following variational system:

$$
\begin{aligned}
& \frac{d(\Delta \mathbf{x}(t))}{d t}=\frac{\partial \mathbf{f}\left(t,\left[\mathbf{x}, \mathbf{y}_{1}, \ldots, \mathbf{y}_{m_{1}}, \mathbf{u}, \mathbf{v}_{1}, \ldots, \mathbf{v}_{m_{2}}\right](t)\right)}{\partial \mathbf{x}} \Delta \mathbf{x}(t) \\
& +\frac{\partial \mathbf{f}\left(t,\left[\mathbf{x}, \mathbf{y}_{1}, \ldots, \mathbf{y}_{m_{1}}, \mathbf{u}, \mathbf{v}_{1}, \ldots, \mathbf{v}_{m_{2}}\right](t)\right)}{\partial \mathbf{y}_{1}} \Delta \mathbf{y}_{1}(t) \\
& +\cdots+\frac{\partial \mathbf{f}\left(t,\left[\mathbf{x}, \mathbf{y}_{1}, \ldots, \mathbf{y}_{m_{1}}, \mathbf{u}, \mathbf{v}_{1}, \ldots, \mathbf{v}_{m_{2}}\right](t)\right)}{\partial \mathbf{y}_{m_{1}}} \Delta \mathbf{y}_{m_{1}}(t) \\
& +\frac{\partial \mathbf{f}\left(t,\left[\mathbf{x}, \mathbf{y}_{1}, \ldots, \mathbf{y}_{m_{1}}, \mathbf{u}, \mathbf{v}_{1}, \ldots, \mathbf{v}_{m_{2}}\right](t)\right)}{\partial \mathbf{u}} \Delta \mathbf{u}(t) \\
& +\frac{\partial \mathbf{f}\left(t,\left[\mathbf{x}, \mathbf{y}_{1}, \ldots, \mathbf{y}_{m_{1}}, \mathbf{u}, \mathbf{v}_{1}, \ldots, \mathbf{v}_{m_{2}}\right](t)\right)}{\partial \mathbf{v}_{1}} \Delta \mathbf{v}_{1}(t) \\
& +\cdots+\frac{\partial \mathbf{f}\left(t,\left[\mathbf{x}, \mathbf{y}_{1}, \ldots, \mathbf{y}_{m_{1}}, \mathbf{u}, \mathbf{v}_{1}, \ldots, \mathbf{v}_{m_{2}}\right](t)\right)}{\partial \mathbf{v}_{m_{2}}} \Delta \mathbf{v}_{m_{2}}(t),
\end{aligned}
$$

with

$$
\begin{aligned}
& \Delta \mathbf{x}(t)=0, \quad t \leq 0, \\
& \Delta \mathbf{u}(t)=0, \quad t<0 .
\end{aligned}
$$


To proceed further, let us express $g_{i}(\mathbf{u})$ as

$$
g_{i}(\mathbf{u})=\Phi_{i}(\mathbf{x}(T))+\int_{0}^{T} \mathcal{L}_{i}\left(\left[\mathbf{x}, \mathbf{y}_{1}, \ldots, \mathbf{y}_{m_{1}}, \mathbf{u}, \mathbf{v}_{1}, \ldots, \mathbf{v}_{m_{2}}\right](t)\right) d t
$$

Define

$$
\begin{aligned}
& \overline{\mathcal{L}}_{i}= \mathcal{L}_{i}\left(t,\left[\mathbf{x}, \mathbf{y}_{1}, \ldots, \mathbf{y}_{m_{1}}, \mathbf{u}, \mathbf{v}_{1}, \ldots, \mathbf{v}_{m_{2}}\right](t)\right), \\
& \mathcal{L}_{i \alpha_{k}}= \mathcal{L}_{i}\left(t+\alpha_{k}, \mathbf{z}_{k}(t), \mathbf{x}\left(t-\alpha_{1}+\alpha_{k}\right), \ldots, \mathbf{x}\left(t-\alpha_{m_{1}}+\alpha_{k}\right), \mathbf{u}\left(t+\alpha_{k}\right),\right. \\
&\left.\mathbf{u}\left(t-\beta_{1}+\alpha_{k}\right), \ldots, \mathbf{u}\left(t-\beta_{m_{2}}+\alpha_{k}\right)\right), \quad k=1,2, \ldots, m_{1}, \\
& \mathcal{L}_{i \beta_{k}}= \mathcal{L}_{i}\left(t+\beta_{k}, \mathbf{x}\left(t+\beta_{k}\right), \mathbf{x}\left(t-\alpha_{1}+\beta_{k}\right), \ldots, \mathbf{x}\left(t-\alpha_{m_{1}}+\beta_{k}\right),\right. \\
&\left.\quad \mathbf{w}_{k}, \mathbf{u}\left(t-\beta_{1}+\beta_{k}\right), \ldots, \mathbf{u}\left(t-\beta_{m_{2}}+\beta_{k}\right)\right), \quad k=1,2, \ldots, m_{2}, \\
& \overline{\mathbf{f}}=\mathbf{f}\left(t,\left[\mathbf{x}, \mathbf{y}_{1}, \ldots, \mathbf{y}_{m_{1}}, \mathbf{u}, \mathbf{v}_{1}, \ldots, \mathbf{v}_{m_{2}}\right](t)\right), \\
& \mathbf{f}_{\alpha_{k}}=\mathbf{f}\left(t+\alpha_{k}, \mathbf{z}_{k}(t), \mathbf{x}\left(t-\alpha_{1}+\alpha_{k}\right), \ldots, \mathbf{x}\left(t-\alpha_{m_{1}}+\alpha_{k}\right), \mathbf{u}\left(t+\alpha_{k}\right),\right. \\
&\left.\quad \mathbf{u}\left(t-\beta_{1}+\alpha_{k}\right), \ldots, \mathbf{u}\left(t-\beta_{m_{2}}+\alpha_{k}\right)\right), \quad k=1,2, \ldots, m_{2}, \\
& \mathbf{f}_{\beta_{k}}=\mathbf{f}\left(t+\beta_{k}, \mathbf{x}\left(t+\beta_{k}\right), \mathbf{x}\left(t-\alpha_{1}+\beta_{k}\right), \ldots, \mathbf{x}\left(t-\alpha_{m_{1}}+\beta_{k}\right),\right. \\
&\left.\quad \mathbf{w}_{k}, \mathbf{u}\left(t-\beta_{1}+\beta_{k}\right), \ldots, \mathbf{u}\left(t-\beta_{m_{2}}+\beta_{k}\right)\right), \quad k=1,2, \ldots, m_{2}, \\
& \bar{H}_{i}(t)=H_{i}\left(t, \mathbf{x}, \mathbf{y}_{1}, \ldots, \mathbf{y}_{m_{1}}, \mathbf{z}_{1}, \ldots, \mathbf{z}_{m_{1}}, \mathbf{u}, \mathbf{v}_{1}, \ldots, \mathbf{v}_{m_{2}},\right. \\
&\left.\quad \mathbf{w}_{1}, \ldots, \mathbf{w}_{m_{2}}, \lambda^{i}, \lambda_{\alpha_{1}}^{i}, \ldots, \lambda_{\alpha_{m_{1}}}^{i}, \lambda_{\beta_{1}}^{i}, \ldots, \lambda_{\beta_{m_{2}}}^{i}\right) .
\end{aligned}
$$

From (3.7),

$$
\begin{gathered}
\Delta g_{i}(\mathbf{u})=\frac{\partial \Phi_{i}(\mathbf{x}(T))}{\partial \mathbf{x}} \Delta \mathbf{x}(T)+\int_{0}^{T}\left[\frac{\partial \overline{\mathcal{L}}_{i}}{\partial \mathbf{x}} \Delta \mathbf{x}(t)+\frac{\partial \overline{\mathcal{L}}_{i}}{\partial \mathbf{y}_{1}} \Delta \mathbf{y}_{1}(t)+\cdots+\frac{\partial \overline{\mathcal{L}}_{i}}{\partial \mathbf{y}_{m_{1}}} \Delta \mathbf{y}_{m_{1}}(t)\right. \\
\left.+\frac{\partial \overline{\mathcal{L}}_{i}}{\partial \mathbf{u}} \Delta \mathbf{u}(t)+\frac{\partial \overline{\mathcal{L}}_{i}}{\partial \mathbf{v}_{1}} \Delta \mathbf{v}_{1}(t)+\cdots+\frac{\partial \overline{\mathcal{L}}_{i}}{\partial \mathbf{v}_{m_{2}}} \Delta \mathbf{v}_{m_{2}}(t)\right] d t
\end{gathered}
$$

In view of (3.8)-(3.10),

$$
\begin{aligned}
& \int_{0}^{T} \frac{\partial \overline{\mathcal{L}}_{i}}{\partial \mathbf{y}_{k}} \Delta \mathbf{y}_{k}(t) d t=\int_{0}^{T} \frac{\partial \mathcal{L}_{i \alpha_{k}}}{\partial \mathbf{x}} \Delta \mathbf{x}(t) e\left(T-t-\alpha_{k}\right) d t, \quad k=1,2, \ldots, m_{1} \\
& \int_{0}^{T} \frac{\partial \overline{\mathcal{L}}_{i}}{\partial \mathbf{v}_{k}} \Delta \mathbf{v}_{k}(t) d t=\int_{0}^{T} \frac{\partial \mathcal{L}_{i \beta_{k}}}{\partial \mathbf{u}} \Delta \mathbf{u}(t) e\left(T-t-\beta_{k}\right) d t, \quad k=1,2, \ldots, m_{2}
\end{aligned}
$$


Thus, from (3.14)-(3.16), it follows that

$$
\begin{aligned}
\Delta g_{i}(\mathbf{u})= & \frac{\partial \Phi_{i}(\mathbf{x}(T))}{\partial \mathbf{x}} \Delta \mathbf{x}(T)+\int_{0}^{T}\left[\frac{\partial \overline{\mathcal{L}}_{i}}{\partial \mathbf{x}} \Delta \mathbf{x}(t)+\frac{\partial \mathcal{L}_{i \alpha_{1}}}{\partial \mathbf{x}} \Delta \mathbf{x}(t) e\left(T-t-\alpha_{1}\right)\right. \\
& +\cdots+\frac{\partial \mathcal{L}_{i_{\alpha_{m_{1}}}}}{\partial \mathbf{x}} \Delta \mathbf{x}(t) e\left(T-t-\alpha_{m_{1}}\right)+\frac{\partial \overline{\mathcal{L}}_{i}}{\partial \mathbf{u}} \Delta \mathbf{u}(t) \\
& \left.+\frac{\partial \mathcal{L}_{i \beta_{1}}}{\partial \mathbf{u}} \Delta \mathbf{u}(t) e\left(T-t-\beta_{1}\right)+\cdots+\frac{\partial \mathcal{L}_{i \beta_{m_{2}}}}{\partial \mathbf{u}} \Delta \mathbf{u}(t) e\left(T-t-\beta_{m_{2}}\right)\right] d t \\
= & \frac{\partial \Phi_{i}(\mathbf{x}(T))}{\partial \mathbf{x}} \Delta \mathbf{x}(T) \\
& +\int_{0}^{T}\left[\frac{\partial \bar{H}_{i}}{\partial \mathbf{x}} \Delta \mathbf{x}(t)-\left(\lambda^{i}(t)\right)^{\mathrm{T}} \frac{\partial \overline{\mathbf{f}}}{\partial \mathbf{x}} \Delta \mathbf{x}(t)-\left(\lambda_{\alpha_{1}}^{i}(t)\right)^{\mathrm{T}} \frac{\partial \mathbf{f}_{\alpha_{1}}}{\partial \mathbf{x}} \Delta \mathbf{x}(t) e\left(T-t-\alpha_{1}\right)\right. \\
& -\cdots-\left(\lambda_{\alpha_{m_{1}}}^{i}(t)\right)^{\mathrm{T}} \frac{\partial \mathbf{f}_{\alpha_{m_{1}}}}{\partial \mathbf{x}} \Delta \mathbf{x}(t) e\left(T-t-\alpha_{m_{1}}\right)+\frac{\partial \bar{H}_{i}}{\partial \mathbf{u}} \Delta \mathbf{u}(t) \\
& -\left(\lambda^{i}(t)\right)^{\mathrm{T}} \frac{\partial \overline{\mathbf{f}}}{\partial \mathbf{u}} \Delta \mathbf{u}(t)-\left(\lambda_{\beta_{1}}^{i}(t)\right)^{\mathrm{T}} \frac{\partial \mathbf{f}_{\beta_{1}}}{\partial \mathbf{u}} \Delta \mathbf{u}(t) e\left(T-t-\beta_{1}\right) \\
& \left.-\cdots-\left(\lambda_{\beta_{m_{2}}}^{i}(t)\right)^{\mathrm{T}} \frac{\partial \mathbf{f}_{\beta_{m_{2}}}}{\partial \mathbf{u}} \Delta \mathbf{u}(t) e\left(T-t-\beta_{m_{2}}\right)\right] d t .
\end{aligned}
$$

From (3.11)-(3.13),

$$
\int_{0}^{T}\left(\lambda^{i}(t)\right)^{\mathrm{T}} \frac{\partial \overline{\mathbf{f}}}{\partial \mathbf{y}_{k}} \Delta \mathbf{y}_{k}(t) d t=\int_{0}^{T}\left(\lambda_{\alpha_{k}}^{i}(t)\right)^{\mathrm{T}} \frac{\partial \mathbf{f}_{\alpha_{k}}}{\partial \mathbf{x}} \Delta \mathbf{x}(t) e\left(T-t-\alpha_{k}\right) d t
$$

for $k=1,2, \ldots, m_{1}$, and

$$
\int_{0}^{T}\left(\lambda^{i}(t)\right)^{\mathrm{T}} \frac{\partial \overline{\mathbf{f}}}{\partial \mathbf{v}_{k}} \Delta \mathbf{v}_{k}(t) d t=\int_{0}^{T}\left(\lambda_{\beta_{k}}^{i}(t)\right)^{\mathrm{T}} \frac{\partial \mathbf{f}_{\beta_{k}}}{\partial \mathbf{u}} \Delta \mathbf{u}(t) e\left(T-t-\beta_{k}\right) d t
$$

for $k=1,2, \ldots, m_{2}$.

We note that

$$
\begin{aligned}
\Delta g_{i}(\mathbf{u})= & \frac{\partial \Phi_{i}(\mathbf{x}(T))}{\partial \mathbf{x}} \Delta \mathbf{x}(T)+\int_{0}^{T}\left[\frac{\partial \bar{H}_{i}}{\partial \mathbf{x}} \Delta \mathbf{x}(t)+\frac{\partial \bar{H}_{i}}{\partial \mathbf{u}} \Delta \mathbf{u}(t)\right. \\
& -\left(\lambda^{i}(t)\right) \mathrm{T}\left(\frac{\partial \overline{\mathbf{f}}}{\partial \mathbf{x}} \Delta \mathbf{x}(t)+\frac{\partial \overline{\mathbf{f}}}{\partial \mathbf{y}_{1}} \Delta \mathbf{y}_{1}(t)+\cdots+\frac{\partial \overline{\mathbf{f}}}{\partial \mathbf{y}_{m_{1}}} \Delta \mathbf{y}_{m_{1}}(t)\right. \\
& \left.\left.+\frac{\partial \overline{\mathbf{f}}}{\partial \mathbf{u}} \Delta \mathbf{u}(t)+\frac{\partial \overline{\mathbf{f}}}{\partial \mathbf{v}_{1}} \Delta \mathbf{v}_{1}(t)+\cdots+\frac{\partial \overline{\mathbf{f}}}{\partial \mathbf{v}_{m_{2}}} \Delta \mathbf{v}_{m_{2}}(t)\right)\right] d t
\end{aligned}
$$

Thus, it follows from (3.17) and (3.4)-(3.6) that

$$
\Delta g_{i}(\mathbf{u})=\frac{\partial \Phi_{i}(\mathbf{x}(T))}{\partial \mathbf{x}} \Delta \mathbf{x}(T)+\int_{0}^{T}\left[\frac{\partial \bar{H}_{i}}{\partial \mathbf{x}} \Delta \mathbf{x}(t)+\frac{\partial \bar{H}_{i}}{\partial \mathbf{u}} \Delta \mathbf{u}(t)-\left(\lambda^{i}(t)\right)^{\mathrm{T}} \frac{d(\Delta \mathbf{x}(t))}{d t}\right] d t
$$


Applying integration by parts to the last term of the right-hand side of the above equation yields

$$
\begin{aligned}
\Delta g_{i}(\mathbf{u})= & \frac{\partial \Phi_{i}(\mathbf{x}(T))}{\partial \mathbf{x}} \Delta \mathbf{x}(T) \\
& +\int_{0}^{T}\left[\frac{\partial \bar{H}_{i}}{\partial \mathbf{x}} \Delta \mathbf{x}(t)+\frac{\partial \bar{H}_{i}}{\partial \mathbf{u}} \Delta \mathbf{u}(t)+\Delta \mathbf{x}(t) \frac{d\left(\lambda^{i}(t)\right)^{\mathrm{T}}}{d t}\right] d t-\left.\lambda^{i}(t)^{\mathrm{T}} \Delta \mathbf{x}(t)\right|_{0} ^{T} .
\end{aligned}
$$

From (3.1), (3.2) and $\Delta \mathbf{x}(0)=0$,

$$
\Delta g_{i}(\mathbf{u})=\int_{0}^{T}\left[\frac{\partial \bar{H}_{i}}{\partial \mathbf{u}} \Delta \mathbf{u}(t)\right] d t .
$$

This completes the proof.

3.2. Approximate problem To construct a sequence of approximate problems by using the control parameterization method [17], let the time interval $[0, T]$ be partitioned into $p$ subintervals $\left[\tau_{j-1}, \tau_{j}\right)$, where $j=1, \ldots, p$. Let the control $\mathbf{u}$ be approximated as a piecewise constant function with possible discontinuities at the time points $\tau_{j}, j=0,1, \ldots, p-1$, given by

$$
\mathbf{u}^{p}(t)= \begin{cases}\gamma(t) & \text { if } t \in\left[-\beta_{m_{2}}, 0\right) \\ \sum_{j=1}^{p} \sigma^{p, j} \chi_{\left[\tau_{j-1}, \tau_{j}\right)}(t) & \text { if } t \in[0, T],\end{cases}
$$

where $\gamma(t)$ is a given piecewise continuous function from $\left[-\beta_{m_{2}}, 0\right)$ into $\mathbb{R}^{r}$ and $\chi_{\left[\tau_{j-1}, \tau_{j}\right)}$ denotes the indicator function of the interval $\left[\tau_{j-1}, \tau_{j}\right.$ ), defined for an interval $I$ by

$$
\chi_{I}(t)= \begin{cases}1 & \text { if } t \in I \\ 0 & \text { otherwise }\end{cases}
$$

Let $\mathcal{U}^{p}$ be the set of all such piecewise constant controls. The function $\mathbf{u}^{p}$ is determined uniquely by the control parameter $\sigma^{p}=\left[\left(\sigma^{p, 1}\right)^{\mathrm{T}}, \ldots,\left(\sigma^{p, p}\right)^{\mathrm{T}}\right]^{\mathrm{T}}$, where $\boldsymbol{\sigma}^{p, j}=\left[\sigma_{1}^{p, j}, \ldots, \sigma_{r}^{p, j}\right]^{\mathrm{T}}, j=1, \ldots, p$. Furthermore, $\boldsymbol{\sigma}^{p, j} \in \mathcal{U}, j=1, \ldots, p$. Let $\Omega$ be the set in $\mathbb{R}^{r p}$ such that $\sigma^{p} \in \Omega$. Thus, Problem $(\mathrm{P} 2(\varepsilon, \delta))$ is approximated as Problem $(\mathrm{P} 2(\varepsilon, \delta)(p))$ given below.

Problem $(\mathrm{P} 2(\varepsilon, \delta)(p))$. Find a control vector $\boldsymbol{\sigma}^{p} \in \Omega$ such that the cost function

$$
\begin{gathered}
\tilde{g}_{0}\left(\sigma^{p}\right)=\Phi_{0}(\mathbf{x}(T))+\int_{0}^{T} \tilde{\mathcal{L}}_{0}\left(t, \mathbf{x}(t), \mathbf{x}\left(t-\alpha_{1}\right), \ldots, \mathbf{x}\left(t-\alpha_{m_{1}}\right),\right. \\
\left.\mathbf{u}^{p}(t), \mathbf{u}^{p}\left(t-\beta_{1}\right), \ldots, \mathbf{u}^{p}\left(t-\beta_{m_{2}}\right)\right) d t
\end{gathered}
$$

is minimized subject to the canonical inequality constraints

$$
\tilde{g}_{i, \varepsilon}\left(\boldsymbol{\sigma}^{p}\right)=\delta+\int_{0}^{T} \tilde{\mathcal{L}}_{i, \varepsilon}\left(t, \mathbf{x}\left(t \mid \boldsymbol{\sigma}^{p}\right)\right) d t \geq 0, \quad i=1, \ldots, N,
$$


where

$$
\tilde{\mathcal{L}}_{i, \varepsilon}\left(t, \mathbf{x}\left(t \mid \boldsymbol{\sigma}^{p}\right)\right)= \begin{cases}h_{i} & \text { if } h_{i}<-\varepsilon \\ -\left(h_{i}-\varepsilon\right)^{2} / 4 \varepsilon & \text { if }-\varepsilon \leq h_{i} \leq \varepsilon \\ 0 & \text { if } h_{i}>\varepsilon,\end{cases}
$$

where $\tilde{\mathcal{L}}_{0}$ is obtained from $\mathcal{L}_{0}$.

To solve Problem $(\mathrm{P} 2(\varepsilon, \delta)(p))$, we need the following theorem which follows readily from Theorem 3.3 .

Theorem 3.4. For each $i=0,1, \ldots, N$ and each $j=1, \ldots, p$, the gradient of the function $\tilde{g}_{i}\left(\sigma^{p}\right)$ with respect to $\sigma^{p, j}$ is

$$
\frac{\partial \tilde{g}_{i}\left(\boldsymbol{\sigma}^{p}\right)}{\partial \boldsymbol{\sigma}^{p, j}}=\int_{I} \frac{\partial \bar{H}_{i}(t)}{\partial \mathbf{u}} d t .
$$

Thus, the approximate problem $(\mathrm{P} 2(\varepsilon, \delta)(p))$ can be solved as a finite-dimensional optimization problem. The following theorem shows that the solution of Problem $(\mathrm{P} 2(\varepsilon, \delta)(p))$ with $\delta>\delta(\varepsilon)$ satisfies the continuous inequality constraints (2.5) if $\delta>0$ is chosen sufficiently small. Its proof is given by Teo et al. [17, Section 8.3].

TheOREM 3.5. For each $\varepsilon>0$, there exists $\delta(\varepsilon)>0$ such that the solution of Problem $(\mathrm{P} 2(\varepsilon, \delta)(p))$ with $\delta>\delta(\varepsilon)$ satisfies the continuous inequality constraints (2.5).

The relationship between Problem $(\mathrm{P} 2(\varepsilon, \delta)(p))$ and Problem $(\mathrm{P} 2(\varepsilon, \delta))$ is furnished in the following two theorems. Proofs of these results are given by Teo et al. [17, Section 8.5]. However, the following condition is assumed:

(A5) For any control $\mathbf{u} \in \mathcal{U}$ such that, for each $i=1, \ldots, N$,

$$
h_{i}(t, \mathbf{x}(t \mid \mathbf{u})) \geq 0 \text { for all } t \in[0, T],
$$

there exists a control $\overline{\mathbf{u}} \in \mathcal{U}$ satisfying, for each $i=1, \ldots, N$,

$$
h_{i}(t, \mathbf{x}(t \mid \mathbf{u}))>0 \text { for all } t \in[0, T],
$$

such that, for each $i=1, \ldots, N$,

$$
h_{i}\left(t, \mathbf{x}\left(t \mid \mathbf{u}^{\alpha}\right)\right)>0 \quad \text { for all } t \in[0, T] \text { and all } \alpha \in(0,1],
$$

where

$$
\mathbf{u}^{\alpha}=\alpha \overline{\mathbf{u}}+(1-\alpha) \mathbf{u} .
$$

We now present the two convergence results in the following two theorems. Here Problem $(\mathrm{P} 2(p))$ is the approximation of Problem $(\mathrm{P} 2)$ with the control $\mathbf{u}$ approximated as a piecewise constant function.

Theorem 3.6. Let $\mathbf{u}_{\varepsilon, \bar{\delta}(\varepsilon)}^{p, *}$ be the optimal control of Problem $(\mathrm{P} 2(\varepsilon, \bar{\delta}(\varepsilon))(p))$, where $\bar{\delta}(\varepsilon)$ is chosen such that the continuous inequality constraints (2.5) are satisfied. Then

$$
g_{0}\left(\mathbf{u}_{\varepsilon, \bar{\delta}(\varepsilon)}^{p, *}\right) \rightarrow g_{0}\left(\mathbf{u}^{p, *}\right)
$$

as $\varepsilon \rightarrow 0$, where $\mathbf{u}^{p, *}$ is the optimal control of Problem $(\mathrm{P} 2(p))$. 
Theorem 3.7. Let $\mathbf{u}^{p, *}$ be the optimal control of Problem $(\mathrm{P} 2(p))$. Then

$$
g_{0}\left(\mathbf{u}^{p, *}\right) \rightarrow g_{0}\left(\mathbf{u}^{*}\right) \text { as } p \rightarrow \infty .
$$

3.3. Solution algorithm Now, for a given parameter $\sigma^{p, j}, j=1, \ldots, p$, the values of the cost and constraint functions and the corresponding gradients with respect to the control parameters can be calculated as detailed in the following algorithm. However, it should be noted that by virtue of the presence of multiple time delays in the system dynamics, the state and co-state systems should be solved as described in Remarks 3.1 and 3.2 , respectively.

Algorithm 3.8. Input parameters $\varepsilon>0, \delta>0$.

1. For each given $\boldsymbol{\sigma}^{p} \in \Omega$, compute the solution $\mathbf{x}\left(\cdot \mid \boldsymbol{\sigma}^{p}\right)$ of the multiple-time delayed system (2.1)-(2.2) by solving the differential equations (2.1) forward in time from $t=0$ to $t=T$ with the initial conditions (2.2).

2. Compute the values of the cost function $\tilde{g}_{0}\left(\sigma^{p}\right)$ and constraint function $\tilde{g}_{i}\left(\sigma^{p}\right)$ for each $i=1, \ldots, N$.

3. Compute the solution $\lambda^{i}\left(\cdot \mid \sigma^{p}\right)$ of the co-state system (3.1)-(3.3) by solving the differential equations (3.1) backward in time from $t=T$ to $t=0$ with the boundary conditions (3.2) and (3.3).

4. The gradients of the cost function $\tilde{g}_{0}\left(\boldsymbol{\sigma}^{p}\right)$ and constraint function $\tilde{g}_{i}\left(\boldsymbol{\sigma}^{p}\right), i=$ $1, \ldots, N$, with respect to $\sigma^{p}$ can be computed according to (3.19).

5. Solve the approximate Problem $(\mathrm{P} 2(\varepsilon, \delta)(p))$ by using the SQP approximation scheme. Let the optimal solution obtained be denoted by $\sigma_{\varepsilon, \delta}^{p, *}$.

6. Check the feasibility of $h_{i}\left(t, \mathbf{x}\left(t \mid \sigma_{\varepsilon, \delta}^{p, *}\right)\right) \geq 0, i=1, \ldots, N$, for all $t \in[0, T]$. If $\sigma_{\varepsilon, \delta}^{p, *}$ is feasible, go to step 8; otherwise, go to step 7 .

7. Set $\delta=\delta / 10$ and go to step 1 .

8. Set $\varepsilon=\varepsilon / 10$. If $\varepsilon>\varepsilon_{\min }$, go to step 1 ; otherwise successfully exit.

\section{Numerical simulation}

Consider an optimal control problem where zinc powder consumption is to be minimized in the zinc sulphate electrolyte purification process of removing cobalt and cadmium ions, the two main metallic ion impurities when zinc powder is present in the reaction tank [21]. The dynamical model for the purification process involves multiple time delays in the state and the control input. In a practical industrial process, it usually takes some time for zinc sulphate electrolyte to flow from the inlet to the outlet of the reaction tank. From analysis of the production data, the duration time of the zinc sulphate solution in the purification reaction tank is mainly affected by the current flow. Although the flow will stabilize at around $200 \mathrm{~m}^{3} \mathrm{~h}^{-1}$, there is some fluctuation within certain bounds. Furthermore, different flows will cause different time delays. More specifically, there are two kinds of delay: 1 hour and 2 hours. Since the addition of zinc powder is adjusted at each hour according to the cobalt and cadmium ion concentrations, there exists a delay of 1 hour in the control. Thus, 
this is a multiple-time delayed reaction process. Owing to the complicated reaction environment and uncertainties, the zinc powder is added much more excessively than required so as to guarantee the quality of the production. Hence, a large amount of zinc powder is wasted. Clearly, this is not desirable in practice.

The reaction process occurs in an ideal continuous stirred tank reactor (CSTR). Furthermore, based on the replacement reaction of the metallic ions, the chemical reaction can be described by the equations

$$
\begin{aligned}
& V \frac{d x_{1}(t)}{d t}=Q x_{10}-Q x_{1}(t-2)-k_{1} U_{1}(t-1) x_{1}(t-1)+\alpha x_{2}(t-2), \\
& V \frac{d x_{2}(t)}{d t}=Q x_{20}-Q x_{2}(t-2)-k_{2} U_{2}(t-1) x_{2}(t-1)+\beta x_{1}(t-2),
\end{aligned}
$$

where $x_{10}=0.01 \mathrm{~g} \mathrm{~L}^{-1}, x_{20}=0.1 \mathrm{~g} \mathrm{~L}^{-1}, Q=200 \mathrm{~m}^{3} \mathrm{~h}^{-1}, V=400 \mathrm{~m}^{3}$, and $x_{1}(t-2)$ and $x_{2}(t-2)$ represent, respectively, the concentrations of cobalt and cadmium ions at the outlet of the reaction tank 2 hours earlier. Here $U_{1}(t)$ and $U_{2}(t)$, which are the zinc powder reaction surface areas for the two types of metallic impurity ions, are the control variables. They can be converted into weights of zinc powder because the relationship between reaction surface area and weight is linear (that is, $U=$ $1740\left(\mathrm{~m}^{2} \mathrm{~kg}^{-1}\right) \times G(\mathrm{~kg})$, where $G$ is the weight of zinc powder) [5, 25]. The constants $k_{1}, k_{2}, \alpha$ and $\beta$ all result from experiments: $k_{1}=5.46 \times 10^{-4}$ and $k_{2}=3.66 \times 10^{-4}$ are, respectively, the chemical reaction coefficients for cobalt and cadmium ions; $\alpha=9.54$ and $\beta=1.415 \times 10^{3}$ are coupling coefficients. Let $\bar{x}_{j}(t), j=1,2$, be the concentrations of cobalt and cadmium ions corresponding to the parameters $k_{1}, k_{2}, \alpha$ and $\beta$ that best fit the observed data.

The described problem can be stated as a multiple-time delayed optimal control problem in the form of Problem (P2), where

$$
J_{2}=\left(x_{1}(8)-\bar{x}_{1}(8)\right)^{2}+\left(x_{2}(8)-\bar{x}_{2}(8)\right)^{2}+\int_{0}^{8}\left(U_{1}(t)^{2}+U_{2}(t)^{2}\right) d t
$$

is minimized with respect to the control parameters $\sigma_{1}^{i}$ and $\sigma_{2}^{i}, i=1, \ldots, 8$, subject to the continuous state inequality constraints

$$
g_{j, e}(x)=e-\left(x_{j}(t)-\bar{x}_{j}(t)\right)^{2} \geq 0, \quad t \in[0,8], j=1,2,
$$

where

$$
U_{1}(t)=\left\{\begin{array}{ll}
\gamma(t) & \text { if } t<0 \\
\sum_{i=1}^{8} \sigma_{1}^{i} \chi_{\left[t_{i-1}, t_{i}\right)}(t) & \text { if } t \geq 0,
\end{array} \quad U_{2}(t)= \begin{cases}\gamma(t) & \text { if } t<0 \\
\sum_{i=1}^{8} \sigma_{2}^{i} \chi_{\left[t_{i-1}, t_{i}\right)}(t) & \text { if } t \geq 0\end{cases}\right.
$$

where $\gamma(t)$ and $\chi_{\left[t_{i-1}, t_{i}\right)}(t)$ are defined as in (3.18), $t_{i}=i, i=0,1, \ldots, 8$, and $e>0$ is an error bound taken as $e=10^{-7}$. 


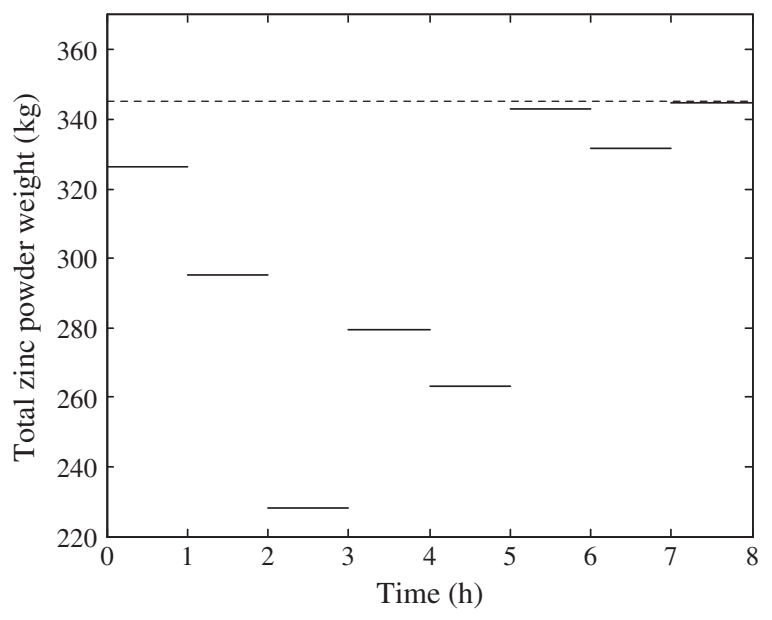

Figure 1. Total zinc powder weight.

With Algorithm 3.8, a gradient-based optimization technique, such as the SQP approximation scheme, can be used to minimize the cost function (4.1) with respect to the control parameters. The states prior to the zero time point are obtained through interpolation of the measured data at the measurement time points prior to the zero time point. The cubic basis spline interpolation method [13] is adopted to construct the fitting curve which passes through all of the measurement data.

The obtained eight optimal control parameters corresponding to $U_{1}$ and $U_{2}$ are given, respectively, by

$$
\begin{array}{llll}
\sigma_{1}^{1}=1.01 \times 10^{5}, & \sigma_{1}^{2}=1.27 \times 10^{5}, & \sigma_{1}^{3}=3.53 \times 10^{4}, & \sigma_{1}^{4}=1.08 \times 10^{5}, \\
\sigma_{1}^{5}=1.04 \times 10^{5}, & \sigma_{1}^{6}=1.10 \times 10^{5}, & \sigma_{1}^{7}=6.58 \times 10^{4}, & \sigma_{1}^{8}=1.08 \times 10^{5}
\end{array}
$$

and

$$
\begin{array}{llll}
\sigma_{2}^{1}=4.67 \times 10^{5}, & \sigma_{2}^{2}=3.86 \times 10^{5}, & \sigma_{2}^{3}=3.62 \times 10^{5}, & \sigma_{2}^{4}=3.78 \times 10^{5}, \\
\sigma_{2}^{5}=3.54 \times 10^{5}, & \sigma_{2}^{6}=4.87 \times 10^{5}, & \sigma_{2}^{7}=5.11 \times 10^{5}, & \sigma_{2}^{8}=4.92 \times 10^{5} .
\end{array}
$$

As mentioned earlier, the amount of zinc powder actually added is expressed as a whole quantity and the reaction surface areas $U_{1}$ and $U_{2}$ can be converted into weight according to the linear relationship between the weight and reaction surface area of zinc powder. Thus, the total weight of zinc powder is as shown in Figure 1. We see that the average weight is much lower than that used in current practice, shown as the dashed line. For the least weight case, it is about $117 \mathrm{~kg}$ lower than that used in current practice. This shows that our proposed optimal control method is effective.

We also obtain the optimal cobalt and cadmium ion concentrations, which are shown as solid lines in Figures 2 and 3. We see that these optimal concentrations remain on track to the measured data. Several measured data deviated rather far away 


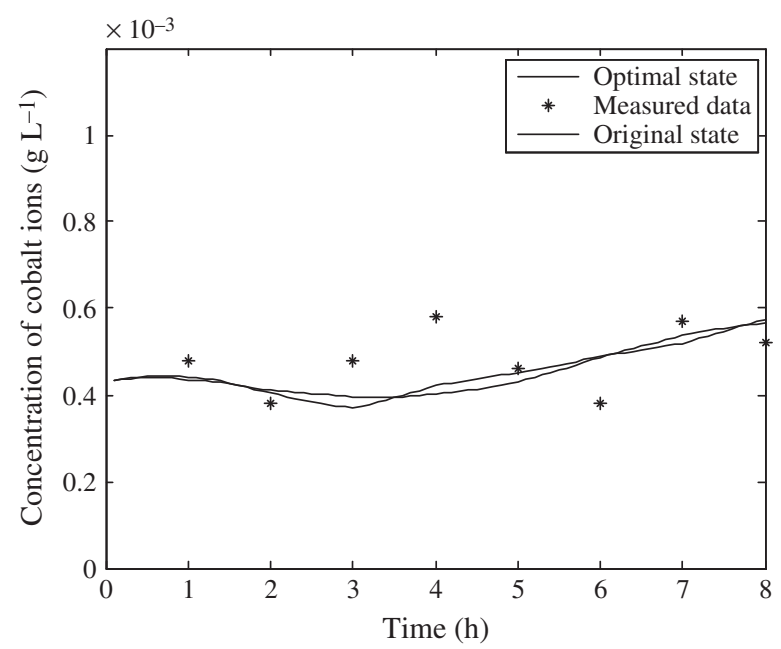

FIGURE 2. Cobalt ion concentration.

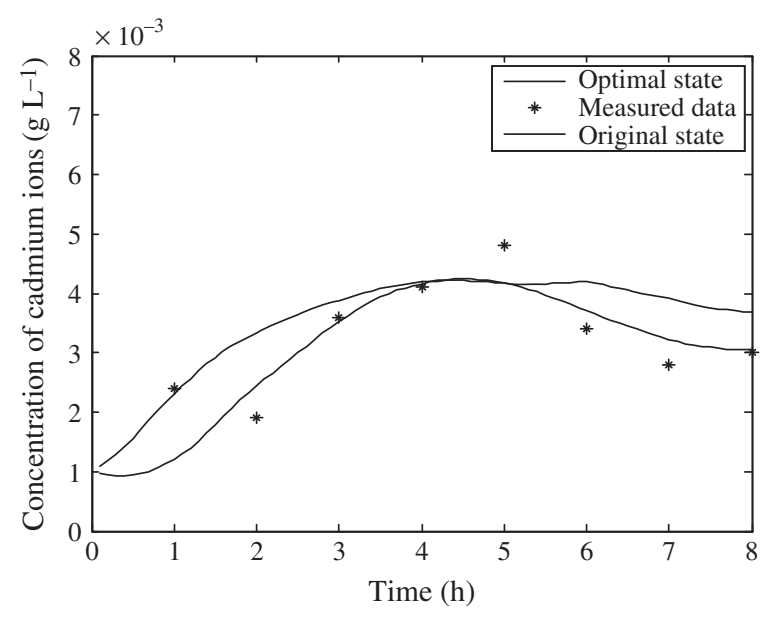

FIGURE 3. Cadmium ion concentration.

from the calculated concentration. This is expected, as the measurements are carried out manually by using some special chemical instruments. Inevitably, this will lead to some measurement inaccuracy. However, the trajectory obtained does reflect the basic characteristic of the practical scenarios under consideration.

\section{Conclusion}

To cater for the presence of multiple time delays in the zinc sulphate electrolyte purification process, a dynamical model described by multiple-time delayed ordinary 
differential equations is constructed. After the system parameters are obtained from chemical experiments, the problem of minimizing the consumption of zinc powder, which is used to remove the metallic ion impurities, can be formulated as an optimal control problem involving a multiple-time delayed dynamical system subject to continuous state inequality constraints. This optimal control problem is then approximated by a sequence of approximate optimal control problems subject to inequality constraints in canonical form. Based on this, the control parameterization method is applied to construct a further sequence of approximate problems. For each approximate problem, gradient formulae for the cost and constraint functions are derived. Hence, this approximate problem can be solved as a nonlinear optimization problem by using the SQP approximation scheme. The numerical results obtained show that the weight of zinc powder is much less than that used in current practice. Thus, the method proposed can reduce the wastage of resources effectively.

\section{Acknowledgements}

The authors would like to thank Professor K. L. Teo with Curtin University of Technology for valuable discussions, and the editors and anonymous reviewers for suggestions and very careful checking that helped to improve the paper. This work was supported in part by the National Natural Science Foundation of China (61074001), in part by the Fundamental Research Funds for the Central Universities (2010QZZD016), in part by the Research Fund for the Doctoral Program of Higher Education of China (20110162130011), and in part by the Program for Changjiang Scholars and Innovative Research Team in University (IRT1044).

\section{References}

[1] M. Basin and J. Rodriguez-Gonzalez, "Optimal control for linear systems with multiple time delays in control input”, IEEE Trans. Automat. Control 51 (2006) 91-97; doi:10.1109/TAC.2005.861718.

[2] L. T. Biegler, "Solution of dynamic optimization problems by successive quadratic programming and orthogonal collocation", Comput. Chem. Eng. 8 (1984) 243-247; doi:10.1016/0098-1354(84)87012-X.

[3] L. T. Biegler and I. E. Grossmann, "Retrospective on optimization", Comput. Chem. Eng. 28 (2004) 1169-1192; doi:10.1016/j.compchemeng.2003.11.003.

[4] B. Chachuat, A. Mitsos and P. I. Barton, "Optimal design and steady-state operation of micro power generation employing fuel cells", Chem. Eng. Sci. 60 (2005) 4535-4556; doi:10.1016/j.ces.2005.02.053.

[5] T. M. Dreher, A. Nelson, G. P. Demopoulos and D. Filippou, "The kinetics of cobalt removal by cementation from an industrial zinc electrolyte in the presence of $\mathrm{Cu}, \mathrm{Cd}, \mathrm{Pb}, \mathrm{Sb}$ and $\mathrm{Sn}$ additives", Hydrometallurgy 60 (2001) 105-116; doi:10.1016/S0304-386X(00)00152-3.

[6] C. Liu, Z. Gong, E. Feng and H. Yin, "Modelling and optimal control for nonlinear multistage dynamical system of microbial fed-batch culture", J. Ind. Manag. Optim. 5 (2009) 835-850; doi:10.3934/jimo.2009.5.835.

[7] R. C. Loxton, K. L. Teo and V. Rehbock, "Optimal control problems with multiple characteristic time points in the objective and constraints", Automatica 44 (2008) 2923-2929;

doi:10.1016/j.automatica.2008.04.011. 
[8] R. C. Loxton, K. L. Teo, V. Rehbock and K. F. C. Yiu, "Optimal control problems with a continuous inequality constraint on the state and the control", Automatica 45 (2009) 2250-2257; doi:10.1016/j.automatica.2009.05.029.

[9] R. Luus, "Optimal control by dynamic programming using accessible grid points and region contraction”, Hungar. J. Ind. Chem. 17 (1989) 523-543.

[10] R. B. Martin and K. L. Teo, Optimal control of drug administration in cancer chemotherapy (World Scientific, Singapore, 1994).

[11] J. Rajesh, K. Gupta, H. S. Kusumakar, V. K. Jayaraman and B. D. Kulkarni, "Dynamic optimization of chemical processes using ant colony framework", Comput. Chem. 25 (2001) 583-595; doi:10.1016/S0097-8485(01)00081-X.

[12] V. Rehbock and I. Livk, "Optimal control of a batch crystallization process", J. Ind. Manag. Optim. 3 (2007) 585-596; doi:10.3934/jimo.2007.3.585.

[13] V. Rehbock, K. L. Teo and L. S. Jennings, "Suboptimal feedback control for a class of nonlinear systems using spline interpolation", Discrete Contin. Dyn. Syst. 1 (1995) 223-236; doi:10.3934/dcds.1995.1.223.

[14] S. Richardson and S. Wang, "The viscosity approximation to the Hamilton-Jacobi-Bellman equation in optimal feedback control: upper bounds for extended domains", J. Ind. Manag. Optim. 6 (2010) 161-175; doi:10.3934/jimo.2010.6.161.

[15] K. Schittkowski, "NLPQLP: A Fortran implementation of a sequential quadratic programming algorithm with distributed and nonmonotone line search-User's guide, version 2.0", University of Bayreuth, 2004.

[16] K. L. Teo and C. J. Goh, "A computational method for combined optimal parameter selection and optimal control problems with general constraints", J. Aust. Math. Soc. Ser. B 30 (1989) 350-364; doi:10.1017/S0334270000006299.

[17] K. L. Teo, C. J. Goh and K. H. Wong, A unified computational approach to optimal control problems (Longman Scientific and Technical, New York, 1991).

[18] K. L. Teo and L. S. Jennings, "Nonlinear optimal control problems with continuous state inequality constraints", J. Optim. Theory Appl. 63 (1989) 1-22; doi:10.1007/BF00940727.

[19] K. L. Teo, L. S. Jennings, H. W. J. Lee and V. Rehbock, "The control parameterization enhancing transform for constrained optimal control problems”, J. Aust. Math. Soc. Ser. B 40 (1999) 314-335; doi:10.1017/S0334270000010936.

[20] K. L. Teo, V. Rehbock and L. S. Jennings, "A new computational algorithm for functional inequality constrained optimization problems", Automatica 29 (1993) 789-792; doi:10.1016/0005-1098(93)90076-6.

[21] L. Y. Wang, W. H. Gui, K. L. Teo, R. C. Loxton and C. H. Yang, "Time delayed optimal control problems with multiple characteristic time points: computation and industrial applications", J. Ind. Manag. Optim. 5 (2009) 705-718; doi:10.3934/jimo.2009.5.705.

[22] K. H. Wong, D. J. Clements and K. L. Teo, "Optimal control computation for nonlinear time-lag systems”, J. Optim. Theory Appl. 47 (1985) 91-107; doi:10.1007/BF00941318.

[23] C. Z. Wu and K. L. Teo, "Global impulsive optimal control computation", J. Ind. Manag. Optim. 2 (2006) 435-450; doi:10.3934/jimo.2006.2.435.

[24] C. Z. Wu, K. L. Teo and V. Rehbock, "Optimal control of piecewise affine systems with piecewise affine state feedback”, J. Ind. Manag. Optim. 5 (2009) 737-747; doi:10.3934/jimo.2009.5.737.

[25] Q. F. Zhang, Y. G. Zhang and Y. H. Guo, "Effect of zinc powder size for cobalt removal from zinc sulphate solution on unit consumption", Nonferrous Smelting 28 (1999) 30-31 (in Chinese). 